\title{
Global rapid-controllability of a class of semilinear evolution equations
}

\section{Chengqiang Wang*}

\section{*Correspondence:} cqwung@foxmail.com

School of Mathematics, Sichuan University, Chengdu, 610064, P.R. China

\section{Springer}

\begin{abstract}
In this paper, we are concerned with a class of control systems governed by semilinear evolution equations. Under appropriate conditions of different types, we prove that the control system, with arbitrarily a priori fixed Lipschitz-continuous nonlinearity, is globally rapidly exact-controllable and approximate-controllable, respectively.
\end{abstract}

MSC: Primary 34K30; secondary 93B05; 93C25

Keywords: semilinear evolution equations; exact-controllability; approximate controllability

\section{Introduction}

Let $H$ and $U$ be real Hilbert spaces, $A$ the infinitesimal generator of a strongly continuous semigroup $\left\{e^{t A}\right\}_{t \in[0, \infty)}$ of bounded linear operators on $H, B \in \mathscr{L}\left(U ;\left[\mathcal{D}\left(A^{*}\right)\right]^{\prime}\right){ }^{\text {ab }}$ Consider the evolution equation/controlled system

$$
\mathbf{y}^{\prime}(t)=A \mathbf{y}(t)+\mathbf{f}(\mathbf{y}(t), t)+B \mathbf{u}(t) \quad \text { for } t \in(0, \infty)
$$

where $\mathbf{u}$ is given in the Fréchet space $L_{\mathrm{loc}}^{2}([0, \infty) ; U)$ and is viewed as a control, and, unless specified otherwise, $\mathbf{f}$ is arbitrarily fixed in the following set:

$$
\begin{aligned}
\mathfrak{L}_{H}:= & \left\{\psi \in \mathscr{C}(H \times[0, \infty)) ; \psi(0, \cdot) \in L_{\text {loc }}^{1}([0, \infty) ; H),\right. \text { and there exists a } \\
& \text { non-decreasing function } \kappa:[0, \infty) \rightarrow(0, \infty) \text { such } \\
& \text { that } \left.\left\|\psi\left(h_{1}, t\right)-\psi\left(h_{2}, t\right)\right\|_{H} \leq \kappa(t)\left\|h_{1}-h_{2}\right\|_{H}, \forall\left(h_{1}, h_{2}\right) \in H^{2}\right\} .
\end{aligned}
$$

To provide the least restrictive condition to guarantee the well-posedness of the initialvalue problem for equation (1.1) (see Definition 1 for the precise sense of well-posedness), we assume hereafter that $B$ satisfies

$$
\exists t \in(0, \infty) \text { s.t. } \int_{0}^{t} e^{(t-\tau) A} B \mathbf{u}(\tau) d \tau \in H, \quad \forall \mathbf{u} \in L_{\mathrm{loc}}^{2}([0, \infty) ; U),
$$

in which the integral is well defined (and hence given initially) in $\left[\mathcal{D}\left(A^{*}\right)\right]^{\prime}$. As stated in Theorem 2.1, the equation/system (1.1) under the condition (1.3) admits a unique solution/trajectory $\mathbf{y} \in \mathscr{C}([0, \infty) ; H)$ with $\mathbf{y}(0)=\mathbf{y}^{0}$ for every pair $\left(\mathbf{y}^{0}, \mathbf{u}\right) \in H \times L_{\text {loc }}^{2}([0, \infty) ; U)$; see Definition 1 for the notion of a solution/trajectory.

(c) The Author(s) 2016. This article is distributed under the terms of the Creative Commons Attribution 4.0 International License (http://creativecommons.org/licenses/by/4.0/), which permits unrestricted use, distribution, and reproduction in any medium, provided you give appropriate credit to the original author(s) and the source, provide a link to the Creative Commons license, and indicate if changes were made. 
The principal concern of this paper is concentrated on the controllability of the system (1.1). Let $T \in(0, \infty)$ be fixed. The system (1.1) is said to be exact-controllable in time $T$ if for every pair $\left(\mathbf{y}^{0}, \mathbf{y}^{T}\right) \in H^{2}$, there exists a control $\mathbf{u} \in L_{\text {loc }}^{2}([0, \infty) ; U)$ such that the unique trajectory $\mathbf{y} \in \mathscr{C}([0, \infty) ; H)$ of the system (1.1) with $\mathbf{y}(0)=\mathbf{y}^{0}$ satisfies $\mathbf{y}(T)=\mathbf{y}^{T}$. The system (1.1) is said to be approximately controllable in time $T$ if for every pair $\left(\mathbf{y}^{0}, \mathbf{y}^{T}\right) \in H^{2}$, for every $\varepsilon \in(0, \infty)$, there exists a control $\mathbf{u} \in L_{\mathrm{loc}}^{2}([0, \infty)$; $U)$ such that the unique trajectory $\mathbf{y} \in \mathscr{C}([0, \infty) ; H)$ of the system (1.1) with $\mathbf{y}(0)=\mathbf{y}^{0}$ satisfies $\left\|\mathbf{y}(T)-\mathbf{y}^{T}\right\|_{H}<\varepsilon$.

The controllability of the system (1.1) in a fixed time $T$ has been studied extensively in the literature. When $\mathbf{f} \equiv 0$, the exact/approximate controllability of the system (1.1) (written in the abstract form) is well understood and has elegant characterizations which are often easy to check; see $[1,2]$ for the details. The idea behind the proof of these characterizations has been used in recent years to check the controllability of systems governed by partial/ordinary differential equations; see [3-6] ([5, 7-20], resp. [6, 21-23]) and the references cited therein for control/observation results concerning systems governed by the 1-D linear hyperbolic systems (say, the transport equation) (heat equation, wave equation, Schrödinger equation, resp. Korteweg-de Vries equation). It is worth pointing out that linear systems governed by 1-D linear hyperbolic systems or linear wave equations (with constant/variable coefficients) are exactly controllable only when the time duration $T$ (given a priori) for control actions is sufficiently long; this phenomenon results from the finite speed of propagation of these systems and can be circumvented by employing controls whose location changes as times evolves (see $[12,13]$ for control results of wave equation with non-cylindrical control domains, and a brief description of the notion of the finite speed of propagation can bee seen in [6]).

When $\mathbf{f} \not \equiv 0$, the system (1.1) (written in the abstract form) has also been investigated extensively for its exact/approximate controllability; see [24-35] and the references therein. Quite recently, approximate controllability has been proved for a large number of abstract control systems involved with fractional derivatives; see [35] and the references therein. Such diverse fixed-point methods as Banach's fixed-point theorem, Schauder's fixed-point theorem, and Schaefer's fixed-point theorem are widely used to obtain the desired control properties; see [26, 29, 36].

The notion pursued here is that of rapid exact-/approximate-controllability. Let us stress that we fixed a priori the time duration $T$ when we speak of the notions of exact- and approximate-controllability; see the above three paragraphs. The system (1.1) is said to be rapidly exact- (resp. approximate-) controllable if it is exact- (resp. approximate-) controllable in any time duration $T \in(0, \infty)$. To obtain a clear picture of this notion, one can consider the system (1.1) in two different situations: (1) $\mathbf{f} \equiv 0, H=\mathbb{R}^{n}, U=\mathbb{R}^{m}$, $A \in \mathbb{R}^{n \times n}, B \in \mathbb{R}^{n \times m}$ where $(m, n) \in \mathbb{N}^{2}$, or (2) $\mathbf{f} \equiv 0, H=L^{2}(0,1), U=\mathbb{R}, A$ is defined by $L^{2}(0,1) \supset\left\{\psi \in H^{1}(0,1) ; \psi(0)=0\right\} \ni \phi \mapsto-\phi^{\prime} \in L^{2}(0,1), B$ is given such that its dual is $\left\{\psi \in H^{1}(0,1) ; \psi(1)=0\right\} \ni \phi \mapsto \phi(0) \in \mathbb{R}$. The system (1.1) in the situation (1) is rapidly exact-controllable if it is exact-controllable in a certain fixed time; this fact can be seen by recalling the Kalman rank criterion for controllability of linear time-invariant finitedimensional systems. The system (1.1) in the situation (2) can be envisaged as a 'realization' of the system,

$$
\begin{cases}\partial_{t} y+\partial_{x} y=0 & \text { in }(0,1) \times(0, \infty), \\ y(0, \cdot)=\mathbf{u} & \text { in }(0, \infty)\end{cases}
$$


where $y=y(x, t)$ and $\mathbf{u} \in L_{\text {loc }}^{2}[0, \infty)$; it is exact-controllable in a fixed time $T_{0}$ if and only if $T_{0} \geq 1$. As with the linear wave equation treated in [1,11], the positive waiting-time phenomenon (i.e., $T_{0} \geq 1$ ) results from the finite speed of propagation; see either [5] or [6], pp. 24-30, for the details.

The purpose of this paper is $(\diamond)$ to provide a sufficient condition that, for every $\mathbf{f} \in \mathfrak{L}_{H}$, the system (1.1) be rapidly exact-and/or approximate-controllable. An obvious way to achieve this goal is (ם) to provide a sufficient condition that the linear system (1.1) with $\mathbf{f} \equiv 0$, be rapidly exact-and/or approximate-controllable, and to prove that such control property can be preserved by the system under nonlinear perturbations belonging to $\mathfrak{L}_{H}$. But the following example indicates that we have to do more in this direction to come up with a desired sufficient condition:

$$
\left.\begin{array}{l}
y_{1}^{\prime}(t)=y_{1}(t)+y_{2}(t)+f_{1}\left(y_{1}(t), y_{2}(t), t\right), \\
y_{2}^{\prime}(t)=y_{2}(t)+f_{2}\left(y_{1}(t), y_{2}(t), t\right)+u(t),
\end{array}\right\} \quad \text { for } t \in[0, \infty) .
$$

The system (1.4), with $\left(f_{1}, f_{2}\right)^{\top} \equiv(0,0)^{\top}$, is rapidly exact-controllable since we have $\operatorname{rank}(B$, $A B)=2$ with $(A, B)$ given by

$$
A=\left(\begin{array}{ll}
1 & 1 \\
0 & 1
\end{array}\right) \text { and } B=\left(\begin{array}{l}
0 \\
1
\end{array}\right)
$$

We can show easily that not all perturbations belonging to $\mathfrak{L}_{\mathbb{R}^{2}}$ renders the system (1.4) to be rapidly exact-controllable. Indeed, let $f_{2} \equiv 0$ and define $f_{1} \in \mathfrak{L}_{\mathbb{R}}$ by

$$
f_{1}\left(y_{1}, y_{2}, t\right)= \begin{cases}-y_{2} & \text { if }\left(y_{1}, y_{2}, t\right) \in \mathbb{R}^{1+1} \times[0,1] \\ (t-2) y_{2} & \text { if }\left(y_{1}, y_{2}, t\right) \in \mathbb{R}^{1+1} \times(1,2), \\ 0 & \text { if }\left(y_{1}, y_{2}, t\right) \in \mathbb{R}^{1+1} \times[2, \infty)\end{cases}
$$

This system is exact-controllable in a fixed time $T_{1}$ (if and) only if $T_{1}>1$. In particular, the system (1.4), with $f_{1} \equiv 0, f_{2}$ given by (1.6), is not rapidly exact-controllable; one can see from the proof of Theorem 2.2 that the reason for the lack of rapid exact-controllability is that the nonlinearity $f_{1}$ is much too intense. The above example shows that the procedure $(\square)$ is insufficient to provide a sufficient condition that the system (1.1) be rapidly exact-

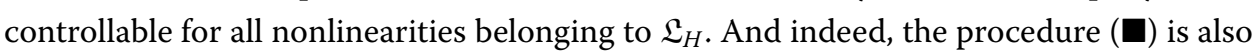
insufficient to provide a desired condition in the approximate-controllability case.

Let us turn to the related references for inspiration. To the best of the author's knowledge, a couple of systems governed by semilinear partial differential equations have already been studied for their rapid (exact and/or approximate) controllability; see $[5,7,8,12,13$, $16,17,21,30,35,37]$ and the references therein.

The route of the study of the rapid approximate-controllability of systems governed by (linear/nonlinear) heat equations is very interesting. Early in 1966, Fattorini [37] showed (among other things) by a Fourier's expansion method that a system described by the 1-D linear heat equation whose Dirichlet boundary value at the right-end point acts as a control is rapidly approximate-controllable; see also [5] and [6], pp. 76-83. After that, the rapid approximate-controllability has been proved for a lot of systems governed by (linear/semilinear) heat equations in the mathematical control community; see [7] and the ref- 
erences therein. Very recently, Bashirov et al. proved that the rapid approximate controllability is preserved for systems subject to bounded nonlinear perturbations; see [38-40]. In [38], Bashirov and Ghahramanlou proved, under the assumptions that the linearized system is rapidly partially approximate-controllable and that the nonlinearity is bounded uniformly, that a class of semilinear systems are rapidly partially approximate-controllable; the assumption on the infinitesimal generator of the dynamics of the systems under consideration is weaker than ours, but the one on the nonlinearity is more stringent than ours. The notion of exact- and approximate partial-controllability were introduced by Bashirov et al. [40], and Bashirov, Etikan and Şemi [41]. See [39] by Bashirov and Ghahramanlou for the counterpart for the stochastic systems.

As indicated previously, thanks to the finite speed of propagation, systems governed by linear wave equations where internal controls and/or boundary controls act on the same 'region' is not rapidly exact-controllable; see [11, 20]. Liu and Yong [12] and Zhang [13] considered systems governed by semilinear wave equations where the control domains vary as time evolves, and they proved under some additional conditions that the systems are rapidly exact-controllable.

From the existing results in the above-mentioned references we can get some useful clues to achieving the goal $(\diamond)$. Let us use the model system (1.1) to introduce the clues. In principle, if the linear system (1.1), with $\mathbf{f} \equiv 0$, is exactly controllable in a fixed time $T_{2}$, then one can prove that the nonlinear system (1.1) is exact-controllable in time $T_{2}$ either by restricting to a subset of the state space or by restricting the intensity of the nonlinearity f. The main tool used in the proof of this principle is the Banach fixed-point theorem. It is interesting to observe that it seems extremely difficult to use the above method to get some elementary results in the rapid approximate-controllability context. What gives us hope is the recent observation stated in [42]: A 'strong' approximate controllability of the linear system (1.1), with $\mathbf{f} \equiv 0$, would support the application of Banach's fixedpoint theorem to obtain approximate controllability results for nonlinear systems with 'weak' nonlinearities or in a certain proper subset of the state space. In the approximatecontrollability part of this paper, we always assume the 'strong' approximate controllability condition on the linear system (1.1) with $\mathbf{f} \equiv 0$; see $(2.8)_{2}$. It is worthy to mention that Zhou [25] provided another condition which guarantees the approximate controllability of the linear system and supports him to prove the approximate controllability of semilinear systems with 'weak' nonlinearity with the help of the approximate controllability of the linear system. It seems more difficult to verify the condition provided by Zhou [25] than the one provided in [42]. Another useful clue is that one can remove both restrictions by assuming and employing compactness.

The first clue helped us a lot in identifying the candidates of the desired condition $(\diamond)$. But we do not go along the second clue to remove the two restrictions mentioned in the previous paragraph; instead, we impose some restrictions on the linear system (1.1), with $\mathbf{f} \equiv 0$, itself so that everyone of its exact-controls (resp. approximate-controls) behaves favorably. The existence of well-behaved exact-controls (resp. approximate-controls) would guarantee the rapid control property of the nonlinear system.

The main results of this paper read as follows. Under the Assumption 1 (resp. Assumption 2), the control system (1.1), with a nonlinearity $\mathbf{f}$ arbitrarily fixed in $\mathfrak{L}_{H}$, is rapidly exact- (resp. approximate-) controllable; see Theorem 2.2 (resp. Theorem 2.3) for the details. 
The rest of the paper is organized as follows. In Section 2 we collect some rudiments necessary for later development, and state explicitly our main results. In Section 3, we prove the rapid exact-controllability part of our main results. In Section 4, we prove the rapid approximate-controllability part of our main results. In Section 5 , several semilinear systems are analyzed with the aid of our abstract results.

\section{Preliminaries and statement of the main results}

In this section, we write down several preliminaries which are available in textbooks concerning mathematical control theory and are necessary in the latter presentation, and we state clearly the main results of this paper.

Lemma 2.1 (see $[2,43])$ Let $A$ be given as in the first paragraph of Section 1.

- $A^{*}$ is the infinitesimal generator of a strongly continuous semigroup $\left\{e^{t A^{*}}\right\}_{t \in[0, \infty)}$. Moreover, we have $e^{t A^{*}}=\left(e^{t A}\right)^{*}$ for every $t \in[0, \infty)$.

- There exists a pair $(M, \omega) \in[1, \infty) \times \mathbb{R}$ such that $\left\|e^{t A}\right\|_{\mathscr{L}(H)} \leq M e^{t \omega}$ for every $t \in[0, \infty)$.

Lemma 2.2 (see [2]) The condition (1.3) is equivalent to each of the following conditions:

(1)

$$
\int_{0}^{t} e^{(t-\tau) A} B \mathbf{u}(\tau) d \tau \in H, \quad \forall(t, \mathbf{u}) \in(0, \infty) \times L_{\mathrm{loc}}^{2}([0, \infty) ; U)
$$

(2)

$$
\begin{aligned}
& \exists(C, t) \in(0, \infty)^{2} \text { s.t. } \\
& \quad \int_{0}^{t}\left\|B^{*} e^{(t-\tau) A^{*}} \phi\right\|_{H}^{2} d \tau \leq C\|\phi\|_{H}^{2}, \quad \forall \phi \in \mathcal{D}\left(A^{*}\right) .
\end{aligned}
$$

(3)

$$
\begin{aligned}
& \forall t \in(0, \infty), \exists C \in(0, \infty) \text { s.t. } \\
& \qquad \int_{0}^{t}\left\|B^{*} e^{(t-\tau) A^{*}} \phi\right\|_{H}^{2} d \tau \leq C\|\phi\|_{H}^{2}, \quad \forall \phi \in \mathcal{D}\left(A^{*}\right) .
\end{aligned}
$$

In the terminology of the community of control theory, $B$ verifying (1.3) is said to be an admissible control operator for the semigroup $\left\{e^{t A}\right\}_{t \in[0, \infty)}$. For admissible control operators, we have:

Lemma 2.3 (see [2] and [42]) If B satisfies (1.3), then the mapping

$$
L_{\mathrm{loc}}^{2}([0, \infty) ; U) \times[0, \infty) \ni(\mathbf{u}, t) \longmapsto \int_{0}^{t} e^{(t-\tau) A} B \mathbf{u}(\tau) d \tau \in H
$$

is well defined and jointly continuous. 
Remark 2.1 By Lemma $2.2((2.1)-+(3)$, more precisely), for every $\widehat{T} \in(0, \infty)$, the linear operator

$$
\begin{gathered}
\mathcal{D}\left(A^{*}\right) \\
\text { (with the relative topology) }
\end{gathered} \eta \longmapsto\left[t \mapsto B^{*} e^{(\widehat{T}-t) A^{*}} \eta\right] \in L^{2}(0, \widehat{T} ; H)
$$

is continuous, and, thanks to the fact that $\mathcal{D}\left(A^{*}\right)$ is dense in $H$, it can be extended into a bounded linear operator of $H$ into $L^{2}(0, \widehat{T} ; H)$. Previously and subsequently, we should understand $t \mapsto B^{*} e^{(\widehat{T}-t) A^{*}} \eta$ as the image of $\eta$ under the unique continuous extension of the linear operator (2.2) when it is only clear that $\eta$ belongs to $H$.

Thanks to Lemma 2.2 ((2.1)-(3), more precisely), it is reasonable to associate to each pair $(A, B)$ for which $B$ is an admissible control operator for the semigroup $\left\{e^{t A}\right\}_{t \in[0, \infty)}$ the function $\mathfrak{q}:[0, \infty) \rightarrow[0, \infty)$ by giving explicitly

$$
\mathfrak{q}(t)=\sqrt{\sup _{h \in \mathcal{D}\left(A^{*}\right),\|\eta\|_{H} \leq 1} \int_{0}^{t}\left\|B^{*} e^{(t-\tau) A^{*}} \eta\right\|_{H}^{2} d \tau}, \quad \forall t \in[0, \infty) .
$$

We can show that $\mathfrak{q}$ is non-decreasing and possesses the property

$$
\mathfrak{q}(t)=\sup _{\substack{(\mathbf{u}, t) \in L_{\mathrm{loc}}^{2}([0, \infty) ; U),\|\mathbf{u}\|_{L^{2}(0, t ; l)} \leq 1}}\left\|\int_{0}^{t} e^{(t-\tau) A} B \mathbf{u}(\tau) d \tau\right\|_{H}, \quad \forall t \in[0, \infty) .
$$

To each $\mathbf{g} \in \mathfrak{L}_{H}$ we associate the function $\mathfrak{p}_{\mathbf{g}}^{H}$ which was precisely defined by

$$
\mathfrak{p}_{\mathbf{g}}^{H}(t)=\sup _{\tau \in[0, t]} \sup _{\substack{\left(h_{1}, h_{2}\right) \in H^{2} \\ h_{1} \neq h_{2}}} \frac{\left\|\mathbf{g}\left(h_{1}, \tau\right)-\mathbf{g}\left(h_{2}, \tau\right)\right\|_{H}}{\left\|h_{1}-h_{2}\right\|_{H}}, \quad \forall t \in[0, \infty)
$$

obviously, $\mathfrak{p}_{\mathbf{g}}^{H}$ is non-decreasing.

Now, we are in a position to present the global well-posedness result of the initial-value problem for equation (1.1). By a solution/trajectory of the equation/system (1.1), we mean the following.

Definition 1 Let $\mathbf{u} \in L_{\mathrm{loc}}^{2}([0, \infty) ; U) . \mathbf{y} \in \mathscr{C}([0, \infty) ; H)$ is said to be a mild solution to equation (1.1) if

$$
\mathbf{y}(t)=e^{t A} \mathbf{y}(0)+\int_{0}^{t} e^{(t-\tau) A}[\mathbf{f}(\mathbf{y}(\tau), \tau)+B \mathbf{u}(\tau)] d \tau, \quad \forall t \in(0, \infty)
$$

In the control community, $\mathbf{y}$ is also called a trajectory of the system (1.1).

The afore-mentioned well-posedness results can be stated as follows.

Theorem 2.1 Let $\mathbf{f} \in \mathfrak{L}_{H}$. The initial-value problem for equation (1.1) is globally wellposed. More precisely, we have: 
- If $\mathbf{y} \in \mathscr{C}([0, \infty) ; H)$ is a solution to equation (1.1), then

$$
\begin{aligned}
\|y(t)\|_{H} \leq & M \int_{0}^{t} \mathfrak{p}_{\mathbf{f}}^{H}(\tau) \exp \left(\int_{\tau}^{t}\left[\omega+M \mathfrak{p}_{\mathbf{f}}^{H}\left(\tau^{\prime}\right)\right] d \tau^{\prime}\right)\left[M e^{\omega \tau}\|\mathbf{y}(0)\|_{H}\right. \\
& \left.+\mathfrak{q}(\tau)\|\mathbf{u}\|_{L^{2}(0, \tau ; U)}+M \int_{0}^{\tau} e^{\omega\left(\tau-\tau^{\prime}\right)}\left\|\mathbf{f}\left(0, \tau^{\prime}\right)\right\|_{H} d \tau^{\prime}\right] d \tau \\
& +M e^{\omega t}\|\mathbf{y}(0)\|_{H}+\mathfrak{q}(t)\|\mathbf{u}\|_{L^{2}(0, t ; U)} \\
& +M \int_{0}^{t} e^{\omega(t-\tau)}\|\mathbf{f}(0, \tau)\|_{H} d \tau, \quad \forall t \in[0, \infty)
\end{aligned}
$$

- If $\mathbf{y}_{1}$ and $\mathbf{y}_{2}$ are two solutions to equation (1.1), with $\mathbf{u}=\mathbf{u}_{1}$, and equation (1.1), with $\mathbf{u}=\mathbf{u}_{2}$, respectively, where $\left(\mathbf{u}_{1}, \mathbf{u}_{2}\right) \in L_{\text {loc }}^{2}\left([0, \infty) ; U^{2}\right)$, then

$$
\begin{aligned}
\left\|\left(\mathbf{y}_{1}-\mathbf{y}_{2}\right)(t)\right\|_{H} \leq & M \int_{0}^{t} \mathfrak{p}_{\mathbf{f}}^{H}(\tau) \exp \left(\int_{\tau}^{t}\left[\omega+M \mathfrak{p}_{\mathbf{f}}^{H}\left(\tau^{\prime}\right)\right] d \tau^{\prime}\right) \\
& \times\left[M e^{\omega \tau}\left\|\left(\mathbf{y}_{1}-\mathbf{y}_{2}\right)(0)\right\|_{H}+\mathfrak{q}(\tau)\left\|\mathbf{u}_{1}-\mathbf{u}_{2}\right\|_{L^{2}(0, \tau ; U)}\right] d \tau \\
& +M e^{\omega t}\left\|\left(\mathbf{y}_{1}-\mathbf{y}_{2}\right)(0)\right\|_{H} \\
& +\mathfrak{q}(t)\left\|\mathbf{u}_{1}-\mathbf{u}_{2}\right\|_{L^{2}(0, t ; U)}, \quad \forall t \in[0, \infty) .
\end{aligned}
$$

- For every pair $\left(\mathbf{y}^{0}, \mathbf{u}\right) \in H \times L_{\text {loc }}^{2}([0, \infty) ; U)$, equation (1.1) admits a unique mild solution $\mathbf{y} \in \mathscr{C}([0, \infty) ; H)$ such that $\mathbf{y}(0)=\mathbf{y}^{0}$.

Proof With the help of (2.4), we can complete the proof by utilizing Banach's fixed-point theorem and an argument used frequently to prove the well-posedness of Cauchy problems for ordinary differential equations. The details are omitted here.

We close this section by stating explicitly the main results of this paper. The first result concerns the rapid exact-controllability of the system (1.1) and holds true under the following assumption.

Assumption 1 There exist a $\widetilde{T} \in(0, \infty)$ and a $\gamma \in \mathscr{C}((0, \widetilde{T}] ;(0, \infty))$ such that

$$
\left\{\begin{array}{l}
\liminf _{t \searrow 0}\left\{t[\gamma(t) \mathfrak{q}(t)]^{2}\right\}=0, \\
\int_{0}^{t}\left\|B^{*} e^{(t-\tau) A^{*}} \eta\right\|_{H}^{2} d \tau \geq[\gamma(t)]^{-2}\|\eta\|_{H}^{2}, \quad \forall(\eta, t) \in \mathcal{D}\left(A^{*}\right) \times(0, \widetilde{T}] .
\end{array}\right.
$$

Theorem 2.2 Suppose that Assumption 1 holds true. For every $\mathbf{f} \in \mathfrak{L}_{H}$, the control system (1.1) is rapidly exact-controllable. Equivalently, for every pair $(T, \mathbf{f}) \in(0, \infty) \times \mathfrak{L}_{H}$, the control system (1.1) is exact-controllable in time $T$.

Another main result of this paper is concerned with the rapid approximatecontrollability of the system (1.1) and holds true under the following assumption. 
Assumption 2 There exist a $\widetilde{T} \in(0, \infty)$ and a $\sigma \in \mathscr{C}((0, \widetilde{T}] ;(0, \infty))$ such that

$$
\left\{\begin{array}{l}
\liminf _{t \unlhd 0}\left\{t[\sigma(t) \mathfrak{q}(t)]^{2}\right\}=0 \\
\int_{0}^{t}\left\|B^{*} e^{(t-\tau) A^{*}} \eta\right\|_{H}^{2} d \tau \geq[\sigma(t)]^{-2} \int_{0}^{t}\left\|e^{(t-\tau) A^{*}} \eta\right\|_{H}^{2} d \tau \\
\forall(\eta, t) \in \mathcal{D}\left(A^{*}\right) \times(0, \widetilde{T}]
\end{array}\right.
$$

Theorem 2.3 Suppose that Assumption 2 holds true. For every $\mathbf{f} \in \mathfrak{L}_{H}$, the control system (1.1) is rapidly approximate-controllable. Equivalently, for every pair $(T, \mathbf{f}) \in(0, \infty) \times$ $\mathfrak{L}_{H}$, the control system (1.1) is approximate-controllable in time $T$.

\section{Proof of Theorem 2.2}

Our purpose in this section is to prove Theorem 2.2. Utilizing $(2.7)_{2}$ in Assumption 1, we construct, in the first step, the exact-controllability Gramians of the linear system (1.1), with $\mathbf{f} \equiv 0$, then analyze the obtained Gramians by using (2.7) in Assumption 1, and lastly gather the properties of the Gramians and the fact that the nonlinearity $\mathbf{f}$ belongs to $\mathfrak{L}_{H}$ to obtain the desired rapid exact-controllability of the nonlinear system (1.1). The Banach contraction mapping fixed-point theorem is used in the third step of the afore-mentioned procedure.

We analyze in this paragraph the controllability Gramians of the linear system (1.1) with $\mathbf{f} \equiv 0$. To every $t \in(0, \widetilde{T}]$ we associate the bounded linear operator ${ }_{t} \mathbf{G} \in \mathscr{L}(H)$ which is defined by

$$
\left.{ }_{t} \mathbf{G} \eta_{1}, \eta_{2}\right\rangle_{H}=\int_{0}^{t}\left\langle B^{*} e^{(t-\tau) A^{*}} \eta_{1}, B^{*} e^{(t-\tau) A^{*}} \eta_{2}\right\rangle_{H} d \tau, \quad \forall\left(\eta_{1}, \eta_{2}\right) \in H^{2}
$$

In view of Remark 2.1, the integral in (3.1) makes sense and the linear operator ${ }_{t} \mathbf{G} \in$ $\mathscr{L}(H)$ is indeed bounded. By the Lax-Milgram lemma, we deduce from Assumption 1 (resp. $(2.7)_{2}$ ) that 0 belongs to the resolvent set $\left.\rho{ }_{t} \mathbf{G}\right)$ of ${ }_{t} \mathbf{G}$ and that

$$
\left\{\begin{array}{l}
\left\langle{ }_{t} \mathbf{G} \eta, \eta\right\rangle_{H} \geq[\gamma(t)]^{-2}\|\eta\|_{H}^{2}, \quad \forall \eta \in H, \\
\left\|\left({ }_{t} \mathbf{G}\right)^{-1}\right\|_{\mathscr{L}(H)} \leq[\gamma(t)]^{2}
\end{array}\right.
$$

for every $t \in(0, \widetilde{T}]$.

Proof of Theorem 2.2 Fix arbitrarily $(T, \mathbf{f}) \in(0, \infty) \times \mathfrak{L}_{H}$.

If $\mathfrak{p}_{\mathbf{f}}^{H}(T)=0$, then it suffices to prove the following control system is exact-controllable in time $T$ :

$$
\mathbf{y}^{\prime}(t)=A \mathbf{y}(t)+B \mathbf{u}(t)+\mathbf{f}(0, t) \quad \text { for } t \in(0, \infty)
$$

But this system is indeed exact-controllable in time $T$ since one can construct for every pair $\left(\mathbf{y}^{0}, \mathbf{y}^{T}\right) \in H^{2}$ the control

$$
\mathbf{u}(t)= \begin{cases}B^{*} e^{(T-t) A^{*}}\left({ }_{t} \mathbf{G}\right)^{-1}\left[\mathbf{y}^{T}-e^{T A} \mathbf{y}^{0}-\int_{0}^{T} e^{(T-\tau) A} \mathbf{f}(0, \tau) d \tau\right] & \text { if } t \in[0, T], \\ 0 & \text { if } t \in(T, \infty),\end{cases}
$$


to drive the system (3.3) steering from the state $\mathbf{y}^{0}$ to the state $\mathbf{y}^{T}$ in time $T$. Therefore, the system (1.1) is exact-controllable in time $T$ when $\mathfrak{p}_{\mathbf{f}}^{H}(T)=0$.

Unless stated otherwise, $\mathfrak{p}_{\mathbf{f}}^{H}(T)>0$ is assumed always in the remainder of the proof. From Assumption 1 (esp. $\left.(2.7)_{1}\right)$ and the fact that $\lim _{t \backslash 0}\left(t e^{t|\omega|}\right)=0$ it follows that there exists a $\bar{T} \in(0, \min (T, \widetilde{T}))$ such that

$$
\left\{\begin{array}{l}
\bar{T} e^{\bar{T}|\omega|} \leq \frac{1}{3 M \mathfrak{p}_{\mathrm{f}}^{H}(T)} \\
\bar{T} e^{\bar{T}|\omega|}[\gamma(\bar{T}) \mathfrak{q}(\bar{T})]^{2} \leq \frac{1}{3 M \mathfrak{p}_{\mathbf{f}}^{H}(T)}
\end{array}\right.
$$

Fix arbitrarily $\left(\mathbf{y}^{0}, \mathbf{y}^{T}\right) \in H^{2}$ henceforth.

By Theorem 2.1, equation (1.1), with $\mathbf{u} \equiv 0$, admits a unique solution $\overline{\mathbf{y}} \in \mathscr{C}([0, \infty) ; H)$ such that $\overline{\mathbf{y}}(0)=\mathbf{y}^{0}$.

With the aid of $\overline{\mathbf{y}}, \bar{T}$, and ${ }_{\bar{T}} \mathbf{G}$, we define a mapping $\Gamma$ on the Banach space $\mathscr{C}([T-$ $\bar{T}, T] ; H)$; the value of $\Gamma$ at $\mathbf{y} \in \mathscr{C}([T-\bar{T}, T] ; H)$ is given by

$$
\begin{aligned}
\Gamma \mathbf{y}(t)= & e^{(t-T+\bar{T}) A} \overline{\mathbf{y}}(T-\bar{T})+\int_{T-\bar{T}}^{t} e^{(t-\tau) A} \mathbf{f}(\mathbf{y}(\tau), \tau) d \tau \\
& +\int_{T-\bar{T}}^{t} e^{(t-\tau) A} B B^{*} e^{(T-\tau) A^{*}}\left({ }_{\bar{T}} \mathbf{G}\right)^{-1} \Lambda \mathbf{y} d \tau, \quad \forall t \in[T-\bar{T}, T],
\end{aligned}
$$

where $\Lambda: \mathscr{C}([T-\bar{T}, T] ; H) \rightarrow H$ is a Lipschitz-continuous mapping whose value at $\mathbf{y}$ is given by

$$
\Lambda \mathbf{y}=\mathbf{y}^{T}-e^{\bar{T} A} \overline{\mathbf{y}}(T-\bar{T})-\int_{T-\bar{T}}^{T} e^{(T-s) A} \mathbf{f}(\mathbf{y}(s), s) d s .
$$

We prove in this paragraph that $\Gamma \mathbf{y}(T-\bar{T})=\overline{\mathbf{y}}(T-\bar{T})$ and $\Gamma \mathbf{y}(T)=\mathbf{y}^{T}$ for every $\mathbf{y} \in$ $\mathscr{C}([T-\bar{T}, T] ; H)$. The first equality is obvious, therefore it suffices to prove the second equality. Let $(\mathbf{y}, h) \in \mathscr{C}([T-\bar{T}, T] ; H) \times H$. Thanks to (3.5) and (3.6), we have

$$
\begin{aligned}
\langle\Gamma \mathbf{y}(T), h\rangle_{H} & =\left\langle\mathbf{y}^{T}-\Lambda \mathbf{y}, h\right\rangle_{H}+\left\langle\int_{T-\bar{T}}^{T} e^{(T-\tau) A} B B^{*} e^{(T-\tau) A^{*}}\left({ }_{\bar{T}} \mathbf{G}\right)^{-1} \Lambda \mathbf{y} d \tau, h\right\rangle_{H} \\
& =\left\langle\mathbf{y}^{T}-\Lambda \mathbf{y}, h\right\rangle_{H}+\int_{T-\bar{T}}^{T}\left\langle B^{*} e^{(T-\tau) A^{*}}\left({ }_{\bar{T}} \mathbf{G}\right)^{-1} \Lambda \mathbf{y}, B^{*} e^{(T-\tau) A^{*}} h\right\rangle_{H} d \tau
\end{aligned}
$$

But

$$
\begin{aligned}
\int_{T-\bar{T}}^{T} & \left.\left\langle B^{*} e^{(T-\tau) A^{*}}{ }_{\bar{T}} \mathbf{G}\right)^{-1} \Lambda \mathbf{y}, B^{*} e^{(T-\tau) A^{*}} h\right\rangle_{H} d \tau \\
& \left.=\int_{0}^{\bar{T}}\left\langle B^{*} e^{(\bar{T}-\tau) A^{*}}{ }_{(\bar{T}} \mathbf{G}\right)^{-1} \Lambda \mathbf{y}, B^{*} e^{(\bar{T}-\tau) A^{*}} h\right\rangle_{H} d \tau \\
& =\left\langle{ }_{\bar{T}} \mathbf{G}\left({ }_{\bar{T}} \mathbf{G}\right)^{-1} \Lambda \mathbf{y}, h\right\rangle_{H} \\
& =\langle\Lambda \mathbf{y}, h\rangle_{H} .
\end{aligned}
$$

Therefore,

$$
\langle\Gamma \mathbf{y}(T), h\rangle_{H}=\left\langle\mathbf{y}^{T}-\Lambda \mathbf{y}, h\right\rangle_{H}+\langle\Lambda \mathbf{y}, h\rangle_{H}=\left\langle\mathbf{y}^{T}, h\right\rangle_{H} .
$$


This, together with the fact that $h \in H$ is arbitrarily chosen, implies $\Gamma \mathbf{y}(T)=\mathbf{y}^{T}$. Noting that $\mathbf{y} \in \mathscr{C}([T-\bar{T}, T] ; H)$ is also arbitrarily chosen, the goal of this paragraph is achieved. Let $\left(\mathbf{y}_{1}, \mathbf{y}_{2}\right) \in \mathscr{C}\left([T-\bar{T}, T] ; H^{2}\right)$. We have by (3.5) immediately

$$
\begin{aligned}
& \left\|\Gamma \mathbf{y}_{2}-\Gamma \mathbf{y}_{1}\right\|_{\mathscr{C}([T-\bar{T}, T] ; H)} \\
& \leq \max _{t \in[T-\bar{T}, T]}\left\|\int_{T-\bar{T}}^{t} e^{(t-\tau) A}\left[\mathbf{f}\left(\mathbf{y}_{1}(\tau), \tau\right)-\mathbf{f}\left(\mathbf{y}_{2}(\tau), \tau\right)\right] d \tau\right\|_{H} \\
& \left.\quad+\max _{t \in[T-\bar{T}, T]} \| \int_{T-\bar{T}}^{t} e^{(t-\tau) A} B B^{*} e^{(T-\tau) A^{*}}{ }_{(\bar{T}} \mathbf{G}\right)^{-1}\left(\Lambda \mathbf{y}_{2}-\Lambda \mathbf{y}_{1}\right) d \tau \|_{H} .
\end{aligned}
$$

Combine Lemma 2.1 and the fact that $\mathbf{f}$ belongs to $\mathfrak{L}_{H}$, to continue as follows:

$$
\begin{aligned}
& \max _{t \in[T-\bar{T}, T]}\left\|\int_{T-\bar{T}}^{t} e^{(t-\tau) A}\left[\mathbf{f}\left(\mathbf{y}_{1}(\tau), \tau\right)-\mathbf{f}\left(\mathbf{y}_{2}(\tau), \tau\right)\right] d \tau\right\|_{H} \\
& \quad \leq M \bar{T} \mathfrak{p}_{\mathbf{f}}^{H}(T) e^{\bar{T}|\omega|}\left\|\mathbf{y}_{2}-\mathbf{y}_{1}\right\|_{\mathscr{C}([T-\bar{T}, T] ; H)} \\
& \quad \leq \frac{1}{3}\left\|\mathbf{y}_{2}-\mathbf{y}_{1}\right\|_{\mathscr{C}([T-\bar{T}, T] ; H)},
\end{aligned}
$$

where the ' $\leq$ ' in the third line follows from (3.4) $)_{1}$. And by a change-of-variable, we have

$$
\begin{aligned}
& \int_{T-\bar{T}}^{t} e^{(t-\tau) A} B B^{*} e^{(T-\tau) A^{*}}\left({ }_{\bar{T}} \mathbf{G}\right)^{-1}\left(\Lambda \mathbf{y}_{2}-\Lambda \mathbf{y}_{1}\right) d \tau \\
& \quad=\int_{0}^{t-(T-\bar{T})} e^{\{[t-(T-\bar{T})]-\tau\} A} B B^{*} e^{(\bar{T}-\tau) A^{*}}\left({ }_{\bar{T}} \mathbf{G}\right)^{-1}\left(\Lambda \mathbf{y}_{2}-\Lambda \mathbf{y}_{1}\right) d \tau, \quad \forall t \in[T-\bar{T}, T]
\end{aligned}
$$

Having this at our disposal, we can prove

$$
\begin{aligned}
& \max _{t \in[T-\bar{T}, T]}\left\|\int_{T-\bar{T}}^{t} e^{(t-\tau) A} B B^{*} e^{(T-\tau) A^{*}}\left(\bar{T}_{\bar{T}} \mathbf{G}\right)^{-1}\left(\Lambda \mathbf{y}_{2}-\Lambda \mathbf{y}_{1}\right) d \tau\right\|_{H} \\
& \quad=\max _{t \in[T-\bar{T}, T]}\left\|\int_{0}^{t-(T-\bar{T})} e^{[[t-(T-\bar{T})]-\tau\} A} B B^{*} e^{(\bar{T}-\tau) A^{*}}\left({ }_{\bar{T}} \mathbf{G}\right)^{-1}\left(\Lambda \mathbf{y}_{2}-\Lambda \mathbf{y}_{1}\right) d \tau\right\|_{H} \\
& \leq \mathfrak{q}(\bar{T})\left[\int_{0}^{\bar{T}}\left\|B^{*} e^{(\bar{T}-\tau) A^{*}}\left(\bar{T}_{\bar{T}} \mathbf{G}\right)^{-1}\left(\Lambda \mathbf{y}_{2}-\Lambda \mathbf{y}_{1}\right)\right\|_{H}^{2} d \tau\right]^{\frac{1}{2}} \\
& \leq[\mathfrak{q}(\bar{T})]^{2}\left\|\left(\overline{(}_{\bar{T}} \mathbf{G}\right)^{-1}\left(\Lambda \mathbf{y}_{2}-\Lambda \mathbf{y}_{1}\right)\right\|_{H} \\
& \leq[\gamma(\bar{T}) \mathfrak{q}(\bar{T})]^{2}\left\|\Lambda \mathbf{y}_{2}-\Lambda \mathbf{y}_{1}\right\|_{H},
\end{aligned}
$$

in which the ' $\leq$ ' in the third line follows from (2.3) and (2.4), the ' $\leq$ ' in the fourth line follows from (2.3), and the ' $\leq$ ' in the fifth line follows from (3.2) 2 . Besides, we can easily get

$$
\begin{aligned}
\left\|\Lambda \mathbf{y}_{2}-\Lambda \mathbf{y}_{1}\right\|_{H} & =\left\|\int_{T-\bar{T}}^{T} e^{(T-s) A}\left[\mathbf{f}\left(\mathbf{y}_{1}(s), s\right)-\mathbf{f}\left(\mathbf{y}_{2}(s), s\right)\right] d s\right\|_{H} \\
& \leq M \bar{T} \mathfrak{p}_{\mathbf{f}}^{H}(T) e^{\bar{T}|\omega|}\left\|\mathbf{y}_{2}-\mathbf{y}_{1}\right\|_{\mathscr{C}([T-\bar{T}, T] ; H)}
\end{aligned}
$$


Substitute (3.10) into (3.9), to arrive at

$$
\begin{aligned}
& \max _{t \in[T-\bar{T}, T]}\left\|\int_{T-\bar{T}}^{t} e^{(t-\tau) A} B B^{*} e^{(T-\tau) A^{*}}\left({ }_{\bar{T}} \mathbf{G}\right)^{-1}\left(\Lambda \mathbf{y}_{2}-\Lambda \mathbf{y}_{1}\right) d \tau\right\|_{H} \\
& \quad \leq M \bar{T} \mathfrak{p}_{\mathbf{f}}^{H}(T)[\gamma(\bar{T}) \mathfrak{q}(\bar{T})]^{2} e^{\bar{T}|\omega|}\left\|\mathbf{y}_{2}-\mathbf{y}_{1}\right\|_{\mathscr{C}([T-\bar{T}, T] ; H)} \\
& \quad \leq \frac{1}{3}\left\|\mathbf{y}_{2}-\mathbf{y}_{1}\right\|_{\mathscr{C}([T-\bar{T}, T] ; H)} .
\end{aligned}
$$

Substitute (3.8) and (3.11) into (3.7), to obtain

$$
\left\|\Gamma \mathbf{y}_{2}-\Gamma \mathbf{y}_{1}\right\|_{\mathscr{C}([T-\bar{T}, T] ; H)} \leq \frac{2}{3}\left\|\mathbf{y}_{2}-\mathbf{y}_{1}\right\|_{\mathscr{C}([T-\bar{T}, T] ; H)}
$$

Since $\left(\mathbf{y}_{1}, \mathbf{y}_{2}\right) \in \mathscr{C}\left([T-\bar{T}, T] ; H^{2}\right)$ is arbitrarily chosen, $\Gamma$ is a contraction mapping on $\mathscr{C}([T-\bar{T}, T] ; H)$. Therefore $\Gamma$ admits a unique fixed-point $\tilde{\mathbf{y}} \in \mathscr{C}([T-\bar{T}, T] ; H)$.

Let us define $\breve{\mathbf{u}} \in L_{\text {loc }}^{2}([0, \infty) ; U)$ by

$$
\breve{\mathbf{u}}(t)= \begin{cases}0 & \text { if } t \in[0, T-\bar{T}), \\ \left.B^{*} e^{(T-t) A^{*}}{ }_{{ }_{T}} \mathbf{G}\right)^{-1} \Lambda \widetilde{\mathbf{y}} & \text { if } t \in[T-\bar{T}, T], \\ 0 & \text { if } t \in(T, \infty),\end{cases}
$$

and denote by $\breve{\mathbf{y}}$ the unique solution to equation (1.1), with $\mathbf{u}=\breve{\mathbf{u}}$, such that $\breve{\mathbf{y}}(0)=\mathbf{y}^{0}$. Noting that $\breve{\mathbf{y}}(T)=\widetilde{\mathbf{y}}(T)=\Gamma \widetilde{\mathbf{y}}(T)=\mathbf{y}^{T}$ and recalling that $\left(\mathbf{y}^{0}, \mathbf{y}^{T}\right) \in H^{2}$ is arbitrarily chosen, we have the summarization: The system (1.1) is exact-controllable in time $T$ when $\mathfrak{p}_{\mathbf{f}}^{H}(T)>0$.

Noting that $(T, \mathbf{f}) \in(0, \infty) \times \mathfrak{L}_{H}$ is arbitrarily chosen (see the first paragraph of this proof), we know that the proof is complete.

\section{Proof of Theorem 2.3}

We prove Theorem 2.3 in this section. Our proof is closely related to an argument used in the reference [42] which is, in turn, highly inspired by [24].

To facilitate the presentation of the proof of Theorem 2.3, we introduce an auxiliary tool, namely, the notion of reachable sets. To each triple $\left(t_{0}, t_{1} ; \mathbf{y}^{t_{0}}\right) \in[0, \infty)^{2} \times H$ with $t_{0}<t_{1}$ we associate the set

$$
\begin{aligned}
\mathcal{R}\left(t_{0}, t_{1} ; \mathbf{y}^{t_{0}} ; \mathbf{f}\right):= & \left\{\mathbf{y}^{t_{1}} \in H ; \exists \mathbf{u}_{0} \in L_{\text {loc }}^{2}([0, \infty) ; U) \text { s.t. } \mathbf{y}^{t_{1}}=\mathbf{y}\left(t_{1}\right)\right. \text { where } \\
& \mathbf{y} \in \mathscr{C}\left(\left[t_{0}, \infty\right) ; H\right) \text { is the unique solution to } \\
& \text { the evolution equation } \\
& \mathbf{y}^{\prime}(t)=A \mathbf{y}(t)+\mathbf{f}(\mathbf{y}(t), t)+B \mathbf{u}_{0}(t) \text { for } t \in\left(t_{0}, \infty\right) \\
& \text { such that } \left.\mathbf{y}\left(t_{0}\right)=\mathbf{y}^{t_{0}}\right\} .
\end{aligned}
$$

When $t_{0}=0$, we write $\mathcal{R}\left(t_{1} ; \mathbf{y}^{0} ; \mathbf{f}\right)=\mathcal{R}\left(0, t_{1} ; \mathbf{y}^{0} ; \mathbf{f}\right)$ for short. In terms of the symbol introduced just now, we restate the approximate controllability as follows. The system (1.1) is approximate-controllable in a fixed time $T$ if and only if for every $\mathbf{y}^{0} \in H, \mathcal{R}\left(T ; \mathbf{y}^{0} ; \mathbf{f}\right)$ is dense in $H$. 
Once the notion of reachable sets is introduced, we are in a position to give a complete proof of Theorem 2.3 .

Proof of Theorem $2.3 \operatorname{Fix}(T, \mathbf{f}) \in(0, \infty) \times \mathfrak{L}_{H}$ in an arbitrary way, and fix $\mathbf{y}^{0} \in H$ arbitrarily.

Thanks to $(2.8)_{1}$ in Assumption 2, there exists a $\bar{T} \in(0, T)$ such that

$$
\sqrt{\bar{T}} \sigma(\bar{T}) \mathfrak{q}(\bar{T}) \mathfrak{p}_{\mathbf{f}}^{H}(T) \exp \left(M \bar{T} \mathfrak{p}_{\mathbf{f}}^{H}(T) e^{\bar{T}|\omega|}\right)<1
$$

Equation (1.1), with $\mathbf{u} \equiv 0$, admits a unique solution $\overline{\mathbf{y}} \in \mathscr{C}([0, \infty) ; H)$ such that $\overline{\mathbf{y}}(0)=\mathbf{y}^{0}$. By an argument used in the proof of [42], Theorem 3.1, and utilizing (4.2), we can prove that $\mathcal{R}(T-\bar{T}, T ; \overline{\mathbf{y}}(T-\bar{T}) ; \mathbf{f})$ is dense in $H$. Therefore, to prove that $\mathcal{R}\left(T ; \mathbf{y}^{0} ; \mathbf{f}\right)$ is dense in $H$, it suffices to prove that $\mathcal{R}(T-\bar{T}, T ; \overline{\mathbf{y}}(T-\bar{T}) ; \mathbf{f})$ is contained in $\mathcal{R}\left(T ; \mathbf{y}^{0} ; \mathbf{f}\right)$.

Let $\mathbf{y}^{T} \in \mathcal{R}(T-\bar{T}, T ; \overline{\mathbf{y}}(T-\bar{T}) ; \mathbf{f})$. By the very definition (see (4.1)), there exists a $\mathbf{u}_{0} \in$ $L_{\text {loc }}^{2}([0, \infty) ; U)$ such that $\mathbf{y}^{T}=\check{\mathbf{y}}(T)$ where $\check{\mathbf{y}} \in \mathscr{C}([T-\bar{T}, \infty) ; H)$ is the unique solution to the Cauchy problem

$$
\left\{\begin{array}{l}
\mathbf{y}^{\prime}(t)=A \mathbf{y}(t)+\mathbf{f}(\mathbf{y}(t), t)+B \mathbf{u}_{0}(t) \quad \text { for } t \in(T-\bar{T}, \infty) \\
\mathbf{y}(T-\bar{T})=\overline{\mathbf{y}}(T-\bar{T})
\end{array}\right.
$$

Define $\breve{\mathbf{u}} \in L_{\text {loc }}^{2}([0, \infty) ; U)$ by

$$
\breve{\mathbf{u}}(t)= \begin{cases}0 & \text { if } t \in[0, T-\bar{T}], \\ \mathbf{u}_{0}(t) & \text { if } t \in[T-\bar{T}, \infty),\end{cases}
$$

and denote by $\breve{\mathbf{y}}$ the unique solution to equation (1.1), with $\mathbf{u}=\breve{\mathbf{u}}$, such that $\breve{\mathbf{y}}(0)=\mathbf{y}^{0}$. It is straightforward to show that $\breve{\mathbf{y}}(t)=\check{\mathbf{y}}(t)$ for all $t \in[T-\bar{T}, \infty)$. In particular, we have $\mathbf{y}^{T}=\check{\mathbf{y}}(T)=\breve{\mathbf{y}}(T) \in \mathcal{R}\left(T ; \mathbf{y}^{0} ; \mathbf{f}\right)$. Since $\mathbf{y}^{T}$ is given arbitrarily in $\mathcal{R}(T-\bar{T}, T ; \overline{\mathbf{y}}(T-\bar{T}) ; \mathbf{f})$, $\mathcal{R}(T-\bar{T}, T ; \overline{\mathbf{y}}(T-\bar{T}) ; \mathbf{f})$ is indeed contained in $\mathcal{R}\left(T ; \mathbf{y}^{0} ; \mathbf{f}\right)$.

Since $\mathbf{y}^{0} \in H$ is arbitrarily given, the system (1.1) is approximate-controllable in time $T$. Since $(T, \mathbf{f}) \in(0, \infty) \times \mathfrak{L}_{H}$ is arbitrarily chosen, the proof is complete.

\section{Applications. Examples and counterexamples}

We give three examples in this section to illustrate the way to apply our abstract result to 'detect' whether a semilinear system is rapidly exact-controllable.

Example 1 Consider the control system

$$
y^{\prime}(t)=y(t)+f(y(t), t)+u(t) \quad \text { for } t \in(0, \infty)
$$

in which $u \in L_{\text {loc }}^{2}([0, \infty) ; \mathbb{R})$ is a control, and $y \in \mathscr{C}([0, \infty) ; \mathbb{R})$ is a state trajectory. We prove next that this system satisfies the following.

Claim 5.1 For every $f \in \mathfrak{L}_{\mathbb{R}}$, the control system (5.1) is rapidly exact-controllable. Equivalently, for every pair $(T, f) \in(0, \infty) \times \mathfrak{L}_{\mathbb{R}}$, the control system (5.1) is exact-controllable in time $T$. 
Write the system (5.1) in the abstract form (1.1), to obtain $A=1, B=1$. And therefore,

$$
\int_{0}^{t}\left|B^{*} e^{(t-\tau) A^{*}} \eta\right|^{2} d \tau=\int_{0}^{t} e^{2(t-\tau)} d \tau=\frac{1}{2}\left(e^{2 t}-1\right), \quad \forall(t, \eta) \in[0, \infty) \times\{-1,1\} .
$$

This leads to our setting

$$
\left\{\begin{array}{l}
{[\mathfrak{q}(t)]^{2}=\frac{1}{2}\left(e^{2 t}-1\right), \quad \forall t \in[0, \infty)} \\
{[\gamma(t)]^{2}=\frac{2}{e^{2 t}-1}, \quad \forall t \in(0, \infty)}
\end{array}\right.
$$

Noting $\lim _{t \backslash 0}\left\{t[\gamma(t)]^{2}[\sigma(t)]^{2}\right\}=\lim _{t \backslash 0} t=0$, we deduce Claim 5.1 by Theorem 2.2.

Example 2 In this example we revisit the control system (1.4). In Section 1 (i.e., the introduction of this paper), we observed that there exists nonlinearity $\left(f_{1}, f_{2}\right)^{\top} \in \mathfrak{L}_{\mathbb{R}^{2}}$ such that the system (1.4) is not rapidly exact-controllable. We attempt to give in this example a 'plausible' reason why the system (1.4) has such an unsatisfactory behavior.

The control system (1.4) can be rewritten into the abstract form (1.1) with $A$ and $B$ given by (1.5). Conduct several routine calculations, to get

$$
\left.\begin{array}{l}
\max _{\eta \in \mathbb{R}^{2} \text { with }|\eta|=1} \int_{0}^{t}\left|B^{*} e^{(t-\tau) A^{*}} \eta\right|^{2} d \tau=\frac{1}{2}\left(e^{2 t}-1\right), \\
\min _{\eta \in \mathbb{R}^{2} \text { with }|\eta|=1} \int_{0}^{t}\left|B^{*} e^{(t-\tau) A^{*}} \eta\right|^{2} d \tau=\int_{0}^{t} \tau^{2} e^{2 \tau} d \tau
\end{array}\right\}, \quad \forall t \in[0, \infty) .
$$

By L'Hôpital's rule, we obtain

$$
\lim _{t \searrow 0} \frac{2 \int_{0}^{t} \tau^{2} e^{2 \tau} d \tau}{t\left(e^{2 t}-1\right)}=0 \quad \text { or } \quad \lim _{t \searrow 0} \frac{t\left(e^{2 t}-1\right)}{2 \int_{0}^{t} \tau^{2} e^{2 \tau} d \tau}=+\infty .
$$

This implies $\liminf _{t \backslash 0}\left\{t[\gamma(t)]^{2}[\mathfrak{p}(t)]^{2}\right\}=+\infty$ whenever $\gamma$ validates $(2.7)_{2}$ with $A$ and $B$ given by (1.5). That is, Assumption 1 ((2.7), more precisely) is violated. This is a plausible reason why the system (1.4) is not rapidly exact-controllable for every $\left(f_{1}, f_{2}\right)^{\top} \in \mathfrak{L}_{\mathbb{R}^{2}}$.

Example 3 The third example is concerned with 1-D wave equation. More precisely, we consider here the control system

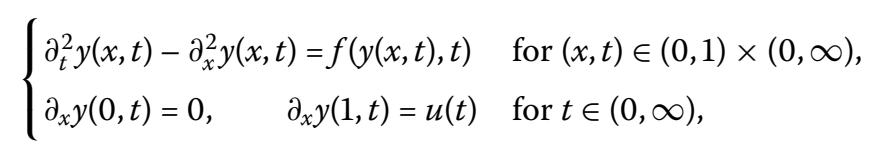

where $u \in L_{\text {loc }}^{2}[0, \infty)$ is a control, $\left(y, \partial_{t} y\right)^{\top} \in \mathscr{C}\left([0, \infty) ; H^{1}(0,1) \times L^{2}(0,1)\right), y=y(x, t)$, is a trajectory, and $f \in \mathfrak{L}_{\mathbb{R}}$.

To write (5.3) into an abstract form like (1.1), we define the state space $H$ by

$$
H:=\left\{\phi \in H^{1}(0,1) ; \int_{0}^{1} \phi(x) d x=0\right\} \times\left\{\phi \in L^{2}(0,1) ; \int_{0}^{1} \phi(x) d x=0\right\},
$$

in which the inner product is given by

$$
H^{2} \ni\left(\left(\begin{array}{l}
\phi_{1} \\
\psi_{1}
\end{array}\right),\left(\begin{array}{l}
\phi_{2} \\
\psi_{2}
\end{array}\right)\right) \longmapsto \int_{0}^{1}\left[\phi_{1}^{\prime}(x) \phi_{2}^{\prime}(x)+\psi_{1}(x) \psi_{2}(x)\right] d x \in \mathbb{R} ;
$$


we write $U=\mathbb{R}$; we define $A$ by

$$
\left\{\begin{array}{l}
\mathcal{D}(A)=\left\{(\phi, \psi)^{\top} \in H ; \phi \in H^{2}(0,1), \phi^{\prime}(0)=\phi^{\prime}(1)=0, \text { and } \psi \in H^{1}(0,1)\right\} \\
A(\phi, \psi)^{\top}=\left(\psi, \phi^{\prime \prime}\right)^{\top}, \quad \forall(\phi, \psi)^{\top} \in \mathcal{D}(A) ;
\end{array}\right.
$$

and we define the control operator $B$ by giving explicitly its adjoint $B^{*}$ as

$$
\mathcal{D}\left(A^{*}\right) \ni(\phi, \psi)^{\top} \longmapsto \psi(1) \in \mathbb{R} .
$$

By simple calculations, $B \alpha=(0, \alpha \delta(x-1))^{\top} \in\left[\mathcal{D}\left(A^{*}\right)\right]^{\prime}$ for every $\alpha \in \mathbb{R}$ where $\delta$ is the Dirichlet measure centered at 0 . Besides, it is well known that $A$ defined by (5.4) is a skew-adjoint operator defined in $H$ (in particular, $\left.\mathcal{D}\left(A^{*}\right)=\mathcal{D}(A)\right)$ and therefore generates thereon a strongly continuous group $\left\{e^{t A}\right\}_{t \in \mathbb{R}}$ of unitary operators.

Thanks to the well-known hidden regularity property, there exists a $C \in(0, \infty)$ such that

$$
\int_{0}^{t}\left|B^{*} e^{(t-\tau) A^{*}} \eta\right|^{2} d \tau \leq C\|\eta\|_{H}^{2}, \quad \forall(\eta, t) \in \mathcal{D}(A) \times[0, \infty) .
$$

On the other hand, it is also well known that

$$
\inf _{\substack{\eta \in \mathcal{D}\left(A^{*}\right),\|\eta\|_{H}=1}}\left[\int_{0}^{t}\left|B^{*} e^{(t-\tau) A^{*}} \eta\right|^{2} d \tau\right]>0 \Longleftrightarrow t \in[2, \infty) .
$$

The proof of (5.5) can be finished via Fourier's series expansion argument and is available in some classical references on control theory; for the sake of completeness, we write it down in detail at the end of this section.

By an argument used to prove [30], Theorem 3.1, we can deduce from (5.5) that, for every $(T, f) \in[2, \infty) \times \mathfrak{L}_{\mathbb{R}}$, the system $(5.3)$ is exact-controllable in time $T$; the compactness contributes in an essential way to guarantee the global exact-controllability for all nonlinearities $f \in \mathfrak{L}_{\mathbb{R}}$.

The system (5.3) is not rapidly exact-controllable for a certain $f \in \mathfrak{L}_{\mathbb{R}}, f \equiv 0$, say. This observation precludes automatically the application of Theorem 2.1 to a judgment of whether the system (5.3) (with a specific nonlinearity) is rapidly exact-controllable. An interesting question yet to be answered is whether the system (5.3) is not rapidly exact-controllable for any nonlinearity $f \in \mathfrak{L}_{\mathbb{R}}$.

To close this section, we would like write down in detail the proof of (5.5).

Proof of (5.5) First of all, it is easy to observe that $t \mapsto e^{(T-t) A^{*}} \eta$ is the solution to the following terminal-boundary value problem:

$$
\begin{cases}\partial_{t}^{2} v-\partial_{x}^{2} v=0 & \text { in }(0,1) \times(0, T) \\ \partial_{x} v(0, \cdot)=\partial_{x} v(1, \cdot)=0 & \text { in }(0, T) \\ v(\cdot, T)=\eta_{1}, \quad \partial_{t} v(\cdot, T)=\eta_{2} & \text { in }(0,1)\end{cases}
$$

where $\left(\eta_{1}, \eta_{2}\right)^{\top}=\eta \in H$. 
Recall that the Fourier cosine series expansion of a function $\psi \in L^{2}(0,1)$ reads

$$
\psi(x)=\sum_{j=0}^{\infty} \widehat{\psi}(j) \cos (j \pi x)
$$

where

$$
\widehat{\psi}(j)=2 \int_{0}^{1} \psi(x) \cos (j \pi x) d x, \quad \forall j \in \mathbb{N}_{0} .
$$

In particular, we have

$$
\eta_{1}(x)=\sum_{j=1}^{\infty} \widehat{\eta_{1}}(j) \cos (j \pi x) \quad \text { and } \quad \eta_{2}(x)=\sum_{j=1}^{\infty} \widehat{\eta_{2}}(j) \cos (j \pi x) .
$$

With the aid of the above expansion, we solve the initial-boundary value problem (5.6), to obtain

$$
v(x, t)=\sum_{j=1}^{\infty}\left\{\widehat{\eta_{1}}(j) \cos [j \pi(T-t)]-\frac{\widehat{\eta_{2}}(j)}{j \pi} \sin [j \pi(T-t)]\right\} \cos (j \pi x),
$$

which implies

$$
\partial_{t} \nu(1, t)=\pi \sum_{j=1}^{\infty} j\left\{\widehat{\eta}_{1}(j) \sin [j \pi(T-t)]+\frac{\widehat{\eta_{2}}(j)}{j \pi} \cos [j \pi(T-t)]\right\} \cos (j \pi) .
$$

If $T \in[2, \infty)$, then we deduce from (5.7) that

$$
\begin{aligned}
& \int_{0}^{T}\left|\partial_{t} v(1, t)\right|^{2} d t \\
& \quad \geq \int_{T-2}^{T}\left|\partial_{t} v(1, t)\right|^{2} d t \\
& \quad=\pi^{2} \int_{T-2}^{T}\left|\sum_{j=1}^{\infty} j\left\{\widehat{\eta_{1}}(j) \sin [j \pi(T-t)]+\frac{\widehat{\eta_{2}}(j)}{j \pi} \cos [j \pi(T-t)]\right\} \cos (j \pi)\right|^{2} d t \\
& =\pi \int_{0}^{2 \pi}\left|\sum_{j=1}^{\infty} j\left[\widehat{\eta_{1}}(j) \sin (t j)+\frac{\widehat{\eta_{2}}(j)}{j \pi} \cos (t j)\right] \cos (j \pi)\right|^{2} d t \\
& =\pi^{2} \sum_{j=1}^{\infty} j^{2}\left(\left|\widehat{\eta}_{1}(j)\right|^{2}+\left|\frac{\widehat{\eta_{2}}(j)}{j \pi}\right|^{2}\right) \\
& \quad=2\left(\left\|\eta_{1}\right\|_{H^{1}(0,1)}^{2}+\left\|\eta_{2}\right\|_{L^{2}(0,1)}^{2}\right)=2\|\eta\|_{H^{2}}^{2} .
\end{aligned}
$$

Write this in terms $A$ and $B$ to get

$$
\inf _{\substack{\eta \in \mathcal{D}\left(A^{*}\right),\|\eta\| H=1}}\left[\int_{0}^{t}\left|B^{*} e^{(t-\tau) A^{*}} \eta\right|^{2} d \tau\right]>0 \Longleftarrow t \in[2, \infty) .
$$


Assume in this paragraph that $T \in(0,2)$. Let $\psi \in \mathscr{C}_{\text {comp }}^{\infty}(0,1 ; \mathbb{R}) \backslash\{\mathbf{0}\}$ with supp $\psi \Subset(0,1-$ $\left.\frac{T}{2}\right)$. Introduce the special function

$$
\bar{v}(x, t)= \begin{cases}\psi\left(x+t-\frac{T}{2}\right) & \text { if }(x, t) \in(0,1) \times\left[\frac{T}{2}, T\right] \\ \psi\left(x-t+\frac{T}{2}\right) & \text { if }(x, t) \in(0,1) \times\left[0, \frac{T}{2}\right]\end{cases}
$$

Obviously, $\bar{v} \in \mathscr{C}\left([0, T] ; H_{0}^{1}(0,1)\right) \cap \mathscr{C}^{1}\left([0, T] ; L^{2}(0,1)\right)$ satisfies the equation (5.6) $)_{1}$. On the other hand, it follows from the condition that $T<2$ that, for $(x, t) \in[0,1] \times[0, T]$,

$$
\begin{aligned}
& t \in\left(\frac{T}{2}, T\right] \text { and } t>x+\frac{T}{2} \quad \Longrightarrow \quad \bar{v}(x, t)=0, \\
& t \in\left(\frac{T}{2}, T\right] \text { and } t<x \quad \Longrightarrow \quad \bar{v}(x, t)=0, \\
& t \in\left[0, \frac{T}{2}\right) \text { and } t<\frac{T}{2}-x \quad \Longrightarrow \quad \bar{v}(x, t)=0, \\
& t \in\left[0, \frac{T}{2}\right) \text { and } t>T-x \quad \Longrightarrow \quad \bar{v}(x, t)=0 .
\end{aligned}
$$

This, together with the fact that supp $\psi \Subset\left(0,1-\frac{T}{2}\right)$, implies $\partial_{x} \bar{v}(0, t)=\partial_{x} \bar{v}(1, t)=\partial_{t} \bar{v}(1, t)=$ 0 for every $t \in[0, T]$. Since $\psi \neq 0$, there exists no $C$ such that

$$
\|\bar{v}(\cdot, T)\|_{H_{0}^{1}(0,1) \times L^{2}(0,1)}^{2} \leq C \int_{0}^{T}\left|\partial_{t} \bar{v}(1, t)\right|^{2} d t .
$$

In other words, the ' $\Rightarrow$ ' part of (5.5) is proved.

To sum up, the proof of (5.5) is complete.

Remark 5.1 Aside from the afore-indicated wave equation and transport equation, there are other systems governed by partial differential equations which display positive waitingtime phenomenon (which also result essentially from the finite speed of propagation). Quite recently, Leiva et al. showed under a certain spectral independence assumption that a broad class of second-order (in time) systems are approximately controllable when the control-acting time is sufficiently long; see $[44,45]$ and the references cited therein.

\section{Conclusion}

By using the usual contraction mapping fixed-point argument and the successive approximation technique developed in [42] (and initially inspired by Zhou [24]), we provide two different quantitative restrictions which are proved to guarantee, respectively, that the exact and approximate controllability is preserved by a class of exactly controllable and approximately controllable systems subject to nonlinear perturbations.

One of the most striking 'peculiarities' is that the perturbations studied here influence the dynamics of the system in a more indirect way. There are indeed perturbations of another type which influence the dynamics of the system in a more immediate way, say the state- and/or control-dependent impulse. Due to its pervasion and wide applicability, control systems subject to nonlinear impulsive perturbations have been studied extensively for their rapid approximate controllability; see [46-53] for rapid approximate-controllability 
results for memoryless semilinear systems, and see $[54,55]$ for results for delayed semilinear systems. Put in an abstract way, Leiva [55] studied the following system for its rapid approximate controllability:

$$
\begin{cases}\mathbf{y}^{\prime}(t)=A \mathbf{y}(t)+\mathbf{f}(\mathbf{y}(t-\tau), \mathbf{u}(t), t)+B \mathbf{u}(t) & \text { for } t \in(0, \infty) \backslash\left(\bigcup_{\ell=1}^{\infty}\left\{t_{\ell}\right\}\right), \\ \mathbf{y}\left(t_{k}\right)=\mathbf{y}\left(t_{k}-0\right)+I_{k}\left(\mathbf{y}\left(t_{k}\right), \mathbf{u}\left(t_{k}\right), t_{k}\right) & \text { for } k \in \mathbb{N}\end{cases}
$$

and Leiva and Sánchez [54] studied the following system for its rapid approximatecontrollability:

$$
\begin{cases}\mathbf{y}^{\prime}(t)=A \mathbf{y}^{\prime}(t)+\beta A \mathbf{y}(t)+\mathbf{f}(\mathbf{y}(t-\tau), \mathbf{u}(t), t)+B \mathbf{u}(t) & \text { for } t \in(0, \infty) \backslash\left(\bigcup_{\ell=1}^{\infty}\left\{t_{\ell}\right\}\right) \\ \mathbf{y}\left(t_{k}\right)=\mathbf{y}\left(t_{k}-0\right)+I_{k}\left(\mathbf{y}\left(t_{k}\right), \mathbf{u}\left(t_{k}\right), t_{k}\right) & \text { for } k \in \mathbb{N}\end{cases}
$$

Inspired by the results of this paper and the ones obtained in the references cited in this section, we are going to investigate the following system for its rapid approximatecontrollability in the near future:

$$
\begin{cases}C \mathbf{y}^{\prime}(t)=D \mathbf{y}(t)+\mathbf{f}(\mathbf{y}(t-\tau), \mathbf{u}(t), t)+B \mathbf{u}(t) & \text { for } t \in(0, \infty) \backslash\left(\bigcup_{\ell=1}^{\infty}\left\{t_{\ell}\right\}\right) \\ \mathbf{y}\left(t_{k}\right)=\mathbf{y}\left(t_{k}-0\right)+I_{k}\left(\mathbf{y}\left(t_{k}\right), \mathbf{u}\left(t_{k}\right), t_{k}\right) & \text { for } k \in \mathbb{N}\end{cases}
$$

The governing equation of the above system is of Sobolev type and has some similarity to the one studied in Ref. [54]. Therefore, it is every interesting to see whether the method used by Leiva and Sanchez [54] can be applied to study the above system.

\section{Competing interests}

The author declares that they have no competing interests.

\section{Author's contributions}

The author contributed to the work totally, and read and approved the final version of the manuscript.

\section{Acknowledgements}

The author is grateful to the reviewers for their valuable suggestions. The author is supported by NSFC (\#11471231, $\# 11571244$ and \#11401404).

\section{Endnotes}

a Throughout the paper, we write $A^{*}$ for the adjoint of $A$ whenever $A: \mathcal{D}(A) \subset H \rightarrow H$ is a densely defined linear operator.

b Let $A: \mathcal{D}(A) \subset H \rightarrow H$ be a densely defined, closed linear operator. $A^{*}$ is also closed and densely defined. Equipped with the inner product $\left(h_{1}, h_{2}\right) \mapsto\left(h_{1}, h_{2}\right)_{H}+\left(A h_{1}, A h_{2}\right)_{H}\left(\operatorname{resp} .\left(h_{1}^{*}, h_{2}^{*}\right) \mapsto\left(h_{1}^{*}, h_{2}^{*}\right)_{H}+\left(A^{*} h_{1}^{*}, A^{*} h_{2}^{*}\right)_{H}\right), \mathcal{D}(A)\left(\operatorname{resp} . \mathcal{D}\left(A^{*}\right)\right)$ is a Hilbert space. $\left[\mathcal{D}\left(A^{*}\right)\right]^{\prime}$ is the topological dual to $\mathcal{D}\left(A^{*}\right)$ with respect to the pivot space $H$. We always identify $\left[\mathcal{D}\left(A^{*}\right)\right]^{\prime \prime}$ with $\mathcal{D}\left(A^{*}\right)$ in the paper. See [43] for a more detailed presentation of the afore-summarized assertions.

Received: 26 January 2016 Accepted: 21 November 2016 Published online: 03 December 2016

\section{References}

1. Lions, J-L: Exact controllability, stabilization and perturbations for distributed systems. SIAM Rev. 30, 1-68 (1988)

2. Tucsnak, M, Weiss, G: Observation and Control for Operator Semigroups. Birkhäuser Advanced Texts: Basler Lehrbücher. Birkhäuser, Basel (2009)

3. Russell, DL: On boundary-value controllability of linear symmetric hyperbolic systems. In: Balakrishnan, AV, Neustadt, LW (eds.) Mathematical Theory of Control, pp. 312-321. Academic Press, New York (1967)

4. Russell, DL: Control theory of hyperbolic equations related to certain questions in harmonic analysis and spectral theory. J. Math. Anal. Appl. 40, 336-368 (1972)

5. Russell, DL: Controllability and stabilizability theory for linear partial differential equations: recent progress and open questions. SIAM Rev. 20, 639-739 (1978)

6. Coron, J-M: Control and Nonlinearity. Mathematical Surveys and Monographs, vol. 136. Am. Math. Soc., Providence (2007) 
7. Zuazua, E: Approximate controllability for semilinear heat equations with globally Lipschitz nonlinearities. Control Cybern. 28, 665-683 (1999)

8. Fursikov, AV, Imanuvilov, OY: Controllability of Evolution Equations. Lecture Notes Series, vol. 34. Seoul National University, Research Institute of Mathematics, Global Analysis Research Center, Seoul (1996)

9. Fernández-Cara, E, Zuazua, E: The cost of approximate controllability for heat equations: the linear case. Adv. Differ. Equ. 5, 465-514 (2000)

10. Fernández-Cara, E, Zuazua, E: Null and approximate controllability for weakly blowing up semilinear heat equations. Ann. Inst. Henri Poincaré, Anal. Non Linéaire 17, 583-616 (2000)

11. Bardos, C, Lebeau, G, Rauch, J: Sharp sufficient conditions for the observation, control, and stabilization of waves from the boundary. SIAM J. Control Optim. 30, 1024-1065 (1992)

12. Liu, K, Yong, J: Rapid exact controllability of the wave equation by controls distributed on a time-variant subdomain. Chin. Ann. Math., Ser. B 20, 65-76 (1999)

13. Zhang, X: Rapid exact controllability of the semilinear wave equation. Chin. Ann. Math., Ser. B 20, 377-384 (1999)

14. Ralston, J: Gaussian beams and the propagation of singularities. In: Littman, W (ed.) Studies in Partial Differential Equations. MAA Stud. Math., vol. 23, pp. 206-248. Math. Assoc. of America, Washington (1982)

15. Maciá, F, Zuazua, E: On the lack of observability for wave equations: a Gaussian beam approach. Asymptot. Anal. 32, $1-26$ (2002)

16. Zuazua, E: Exact boundary controllability for the semilinear wave equation. In: Brézis, H, Lions, J-L (eds.) Nonlinear Partial Differential Equations and Their Applications, pp. 357-391. Longman Sci. Tech, Harlow (1991)

17. Zuazua, E: Exact controllability for semilinear wave equations in one space dimension. Ann. Inst. Henri Poincaré, Anal. Non Linéaire 10, 109-129 (1993)

18. Jaffard, S: Contrôle interne exact des vibrations d'une plaque carrée. C. R. Acad. Sci. Paris Ser. I Math. 307, 759-762 (1988)

19. Lebeau, G: Contrôle de l'equation de Schrödinger. J. Math. Pures Appl. 71, 267-291 (1992)

20. Zuazua, E: Remarks on the controllability of the Schrödinger equation. In: Bandrauk, AD, Delfour, MC, Bris, CL (eds, Quantum Control: Mathematical and Numerical Challenges, pp. 193-211. Am. Math. Soc., Providence (2003)

21. Rosier, L: Exact boundary controllability for the Korteweg-de Vries equation on a bounded domain. ESAIM Control Optim. Calc. Var. 2, 33-55 (1997)

22. Cerpa, E: Control of a Korteweg-de Vries equation: a tutorial. Math. Control Relat. Fields 4, 45-99 (2014)

23. Cerpa, E, Rivas, I, Zhang, B-Y: Boundary controllability of the Korteweg-de Vries equation on a bounded domain. SIAM J. Control Optim. 51, 2976-3010 (2013)

24. Zhou, HX: Approximate controllability for a class of semilinear abstract equations. SIAM J. Control Optim. 21, 551-565 (1983)

25. Zhou, HX: Controllability properties of linear and semilinear abstract control systems. SIAM J. Control Optim. 22, 405-422 (1984)

26. Balachandran, K, Dauer, JP: Controllability of nonlinear systems in Banach spaces: a survey. J. Optim. Theory Appl. 115, 7-28 (2002)

27. Seidman, TI: Invariance of the reachable set under nonlinear perturbations. SIAM J. Control Optim. 25, 1173-1191 (1987)

28. Carmichael, N, Quinn, MD: Fixed-point methods in nonlinear control. IMA J. Math. Control Inf. 5, 41-67 (1988)

29. Dauer, JP, Mahmudov, NI: Controllability of some nonlinear systems in Hilbert spaces. J. Optim. Theory Appl. 123, 319-329 (2004)

30. Zhang, X: Exact controllability of semilinear evolution systems and its application. J. Optim. Theory Appl. 107, 415-432 (2000)

31. Naito, K: Controllability of semilinear control systems dominated by the linear part. SIAM J. Control Optim. 25, 715-722 (1987)

32. Gahl, RD: Controllability of nonlinear systems of neutral type. J. Math. Anal. Appl. 63, 33-42 (1978)

33. Klamka, J: On the global controllability of perturbed nonlinear systems. IEEE Trans. Autom. Control AC-20, 170-172 (1975)

34. Li, XJ, Yong, JM: Optimal Control Theory for Infinite-Dimensional Systems. Systems \& Control: Foundations \& Applications. Birkhäuser, Boston (1995)

35. Hernández, E, O’Regan, D, Balachandran, K: Comments on some recent results on controllability of abstract differential problems. J. Optim. Theory Appl. 159, 292-295 (2013)

36. Balachandran, K, Dauer, JP: Controllability of nonlinear systems via fixed-point theorems. J. Optim. Theory Appl. 53, 345-352 (1987)

37. Fattorini, HO: Control in finite time of differential equations in Banach space. Commun. Pure Appl. Math. 19, 17-34 (1966)

38. Bashirov, AE, Ghahramanlou, N: On partial approximate controllability of semilinear systems. Cogent Eng. 1, Article ID 965947 (2014)

39. Bashirov, AE, Ghahramanlou, N: On partial S-controllability of semilinear partially observable systems. Int. J. Control 88, 969-982 (2015)

40. Bashirov, AE, Mahmudov, N, Semi, N, Etikan, H: Partial controllability concepts. Int. J. Control 80, 1-7 (2007)

41. Bashirov, AE, Etikan, H, Semi, N: Partial controllability of stochastic linear systems. Int. J. Control 83, 2564-2572 (2010)

42. Wang, C: Lipschitz perturbations of a class of approximately controllable linear systems. Adv. Differ. Equ. 2016, Article ID 215 (2016)

43. Pazy, A: Semigroups of Linear Operators and Applications to Partial Differential Equations. Applied Mathematical Sciences, vol. 44. Springer, New York (1983)

44. Leiva, $\mathrm{H}$ : Interior controllability of a broad class of second order equations in $L^{2}(\Omega)$. Rev. Notas Mat. 262, 49-59 (2008)

45. Leiva, $H$, Merentes, N: Controllability of second-order equations in $L^{2}(\Omega)$. Math. Probl. Eng. 2010, Article ID 147195 (2010)

46. Leiva, H: Controllability of semilinear impulsive nonautonomous systems. Int. J. Control 88, 585-592 (2015)

47. Leiva, $\mathrm{H}$, Merentes, N, Sanchez, J: Approximate controllability of a semilinear heat equation. Int. J. Partial Differ. Equ. 2013, Article ID 424309 (2013) 
48. Appell, J, Leiva, $\mathrm{H}$, Merentes, $\mathrm{N}$ : Nonlinear spectral theory and controllability of semilinear evolution equations. Int. J. Evol. Equ. 4, 213-225 (2010)

49. Leiva, H: Rothe's fixed point theorem and controllability of semilinear nonautonomous systems. Syst. Control Lett. 67, 14-18 (2014)

50. Leiva, H: Unbounded perturbation of the controllability for evolution equations. J. Math. Anal. Appl. 280, 1-8 (2003)

51. Leiva, $H$, Nelson, M, Sánchez, JL: Approximate controllability of semilinear reaction diffusion equations. Math. Control Relat. Fields 2, 171-182 (2012)

52. Leiva, H, Merentes, N: Approximate controllability of the impulsive semilinear heat equation. J. Math. Appl. 38, 85-104 (2015)

53. Leiva, H: Approximate controllability of semilinear impulsive evolution equations. Abstr. Appl. Anal. 2015, Article ID 797439 (2015)

54. Leiva, H, Sánchez, JL: Rothe's fixed point theorem and the controllability of the Benjamin-Bona-Mahony equation with impulses and delay. Appl. Math. 7, 1748-1764 (2016)

55. Leiva, $\mathrm{H}$ : Controllability of the semilinear heat equation with impulses and delay on the state. Nonauton. Dyn. Syst. 2, 52-62 (2015)

Submit your manuscript to a SpringerOpen ${ }^{\circ}$ journal and benefit from:

- Convenient online submission

- Rigorous peer review

- Immediate publication on acceptance

- Open access: articles freely available online

- High visibility within the field

Retaining the copyright to your article

Submit your next manuscript at $\boldsymbol{s p r i n g e r o p e n . c o m ~}$ 